\title{
Preface: Special topic on electrocatalysis \& energy science
}

\author{
Changzheng $\mathrm{Wu}^{1,2^{*}}$, Gang $\mathrm{Wu}^{3^{*}} \&$ Guihua $\mathrm{Yu}^{4^{*}}$ \\ ${ }^{1}$ Hefei National Laboratory for Physical Science at the Microscale, CAS Center for Excellence in Nanoscience, iChEM (Collaborative \\ Innovation Center of Chemistry for Energy Materials), University of Science and Technology of China, Hefei 230026, China; \\ ${ }^{2}$ Institute of Energy, Hefei Comprehensive National Science Center, Hefei 230026, China; \\ ${ }^{3}$ Department of Chemical and Biological Engineering, University at Buffalo, The State University of New York, Buffalo, NY 14260, USA; \\ ${ }^{4}$ Materials Science and Engineering, Mechanical Engineering, Texas Materials Institute, University of Texas at Austin, Austin, TX 78712, USA
}

Citation: Wu C, Wu G, Yu G. Preface: Special topic on electrocatalysis \& energy science. Sci China Chem, 2020, 63: 1515-1516, https://doi.org/10.1007/ s11426-020-9894-9

Electrocatalysis is one type of catalysis. The catalysts at the electrode/electrolyte interfaces can facilitate electrochemical reactions with high rates and stability via selective pathways at potentials close to their thermodynamic limits (i.e., minimum overpotentials). The first elaborated study for electrocatalysis can be dated back to the 1920s when Bowden and Rideal examined the electrolytic behaviors of a series of metal films for hydrogen evolution. However, it is not until 1963 that Grubb used the word "electrocatalysis" for the first time to name the electrochemical processes in fuel cells.

Over the last decades, capturing and utilizing transient renewable energies such as solar and wind energy to offset the fast depletion of fossil fuels is becoming one of the mainstreams towards a clean and sustainable energy supply. Among these new energy technologies, energy conversion and storage are the critical steps in which electrocatalysis plays an indispensable role. In contrast to the traditional fossil fuel industry highly relying on high temperature/ pressure reactions, electrocatalysis usually operates at ambient temperature and pressure, significantly reducing largescale investment on the inflexible heat/pressure management equipment. Based on the above considerations and the witness of the growing commercialization of rechargeable battery/fuel cell-powered products, it can be predicted that electrocatalysis-related technologies will dominate the further development in many chemical/energy industries.

*Corresponding authors (email: czwu@ustc.edu.cn; gangwu@buffalo.edu; ghyu@austin.utexas.edu)
So far, the most widely studied electrocatalysis reactions include oxygen evolution reaction (OER), hydrogen evolution reaction (HER), oxygen reduction reaction (ORR), hydrogen oxidation reaction (HOR), $\mathrm{CO}_{2}$ reduction reaction (CRR), nitrogen reduction reaction (NRR), and methanol oxidation reaction (MOR). These electrocatalysis reactions lay the basis for chemicals/fuels production and utilization, e.g., hydrogen production by water splitting, electricity generation via fuel cells, carbon-based chemicals/fuels production, and ammonium electrosynthesis. The rechargeable batteries and supercapacitors involved electrochemical reactions (e.g., oxygen evolution/reduction and metal plating in metal-air batteries and polysulfides redox reactions in Lisulfur batteries) are another essential branch of electrocatalysis which paves the way toward efficient and highdensity energy storage. A third booming development of electrocatalysis is the electrochemical synthesis of organic compounds based on electrocatalytic $\mathrm{C}-\mathrm{H}$ oxidation, $\mathrm{C}=\mathrm{C}$ reduction, dehalogenation reduction, $\mathrm{C}=\mathrm{O}$ reduction, providing alternative and green routes to traditional organic compound synthesis.

In this special topic of the "Science China Chemistry" journal, we collect two reviews, two articles, and one communication to track the most recent progress in electrocatalysis in energy science. The first review by $\mathrm{Hu}$ et al. summarizes the development of $\mathrm{M} / \mathrm{N} / \mathrm{C}$ based non-noble oxygen reduction electrocatalysts from three aspects: (1) strategies for intrinsic activity improvement; (2) porous 
structure engineering; (3) scale-up and cost-effective synthesis of M/N/C electrocatalyst. The second review by Wu et $a l$. highlights the significance of local structure engineering for precious and non-precious metal catalysts for oxygen reduction in proton exchange membrane fuel cells. The first article by Wang et al. reports an $\mathrm{O}$ doped $\mathrm{Ni}_{3} \mathrm{~N}$ electrocatalyst for highly efficient hydrogen evolution in alkali conditions. Both experimental and theoretical investigations reveal the oxygen incorporation facilitates water adsorption and dissociation. The second article by $\mathrm{Yu}$ et al. demonstrates a 3D macroporous carbon-supported $\mathrm{Mo}_{2} \mathrm{C}$ nanodots electrocatalyst for nitrogen reduction with high faradaic ef- ficiency. Detailed electrochemical characterizations reveal the beneficial role of enhanced electron transfer and increased electrochemical surface area from the 3D macroporous carbon framework. Finally, the communication from Wang et al. discloses the detrimental effect of electrolyte additive on $\mathrm{Na}$ metal plating/stripping for thick $\mathrm{Na}$ metal electrodes in rechargeable $\mathrm{Na}$ metal batteries and the importance of strong adhesion between the thin Na layer and $\mathrm{Cu}$ current collector for thin Na metal electrodes. They further introduce a gold interlayer between $\mathrm{Cu}$ current collector and Na layer to realize high-energy-density, high-efficiency, and stable-cycling in $\mathrm{Na}|| \mathrm{Na}_{3} \mathrm{~V}_{2}\left(\mathrm{PO}_{4}\right)_{3}$ batteries.
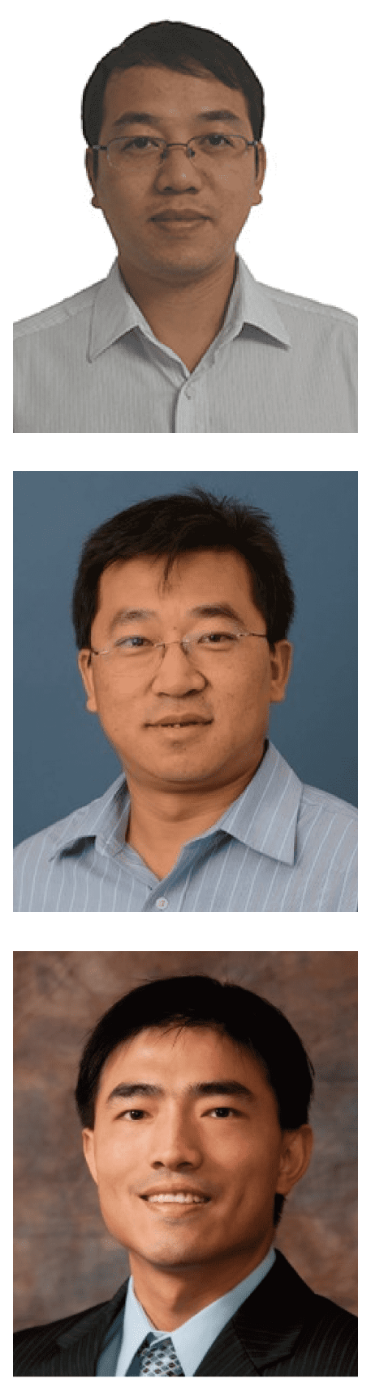

Changzheng Wu obtained his B.S. degree (2002) and his Ph.D. degree (2007) in the Department of Chemistry from the University of Science and Technology of China (USTC). He then worked as a postdoctoral fellow in the Hefei National Laboratory for Physical Sciences at the Microscale. He is now a full professor at the Department of Chemistry, USTC. Prof. Wu's current research interests focus on the synthesis of inorganic 2D nanomaterials, as well as on the regulation of their intrinsic physical properties for wide applications in energy conversion.

Gang $\mathbf{W u}$ is a professor of Chemical Engineering at University at Buffalo (UB), The State University of New York. He obtained his B.S. in 1997 and Ph.D. in 2004 on electrochemical engineering at the Harbin Institute of Technology. Dr. Wu became a staff scientist at Los Alamos National Laboratory (LANL), after completing extensive postdoctoral training at Tsinghua University (2004-2006), the University of South Carolina (2006-2008), and LANL (2008-2010). He joined UB as an assistant professor in 2014 and was quickly promoted as a tenured associate professor in 2018 and a full professor in 2020. His research interests are electrochemical energy science and technology. He is a Highly Cited Researcher ranked by Thomson Reuters, Clarivate Analytics since 2018.

Guihua Yu is a Professor of Materials Science and Mechanical Engineering at University of Texas at Austin. He received his B.S. degree with the highest honor from University of Science and Technology of China and Ph.D. from Harvard University, followed by postdoc at Stanford University. He is a Fellow of Royal Society of Chemistry and Institute of Physics, Sloan Research Fellow, and Camille Dreyfus Teacher-Scholar. His research interests include synthesis and selfassembly of nanoarchitected polymeric materials and hybrid organic-inorganic nanomaterials, fundamental understanding of their chemical/physical properties, and exploration of their technological applications in energy, environment, and sustainability. 